\title{
L'éloquence de la chaire à l'époque romantique : le cas de Lacordaire et de Lamennais
}

\author{
Ecclesiastical rhetoric in the Romantic period: \\ Lacordaire and Lamennais
}

\author{
Marta Sukiennicka \\ Université Adam Mickiewicz de Poznań
}

\begin{abstract}
This paper considers French ecclesiastical rhetoric in the Romantic era, focusing on Lacordaire's and Lamennais' contrasting views on the role of eloquence in religious discourse. It argues that Lacordaire, despite employing secular language in his Conférences remains a classicist religious orator, whereas Lammenais expresses his deep distrust of the spoken word and condemns eloquence, turning to letters and political discourse. The comparison of the two concepts can be seen as an exemplification of the transition from littérature-discours to littérature-texte, a general phenomenon in the French Romantic literature as described by Alain Vaillant.
\end{abstract}

Keywords: French ecclesiastical rhetoric, Romanticism, Lacordaire, Lamennais

Cet article est consacré à la relation entre la rhétorique classique et la prédication chrétienne à l'époque romantique en France. Pendant la période postrévolutionnaire qui signe un certain retour officiel du religieux, l'incompatibilité des sources païennes du modèle de l'éloquence avec le contenu de la doctrine chrétienne commence à être de plus en plus critiquée et peu-à-peu concurrencée par d'autres modes de propagation de la parole sacrée parmi les croyants. Premièrement, nous allons esquisser brièvement l'histoire de cette relation ambiguë entre la rhétorique antique et la prédication chrétienne. Deuxièmement, nous allons poser la question de l'influence des idées romantiques sur la pratique oratoire de la chaire. Dans ce but, nous allons examiner deux modèles de l'éloquence chrétienne à l'époque romantique : 1) celui de l'abbé Lacordaire, auteur des Conférences de Notre-Dame, prononcées dans les années 1835-1854 et provoquant une véritable "bataille romantique de l'éloquence sacrée », selon les mots de Frank Paul Bowman ; 2) celui de Félicité de Lamennais, célèbre auteur du recueil Paroles d'un croyant (1834) qui témoigne déjà d'un certain 
recul par rapport à l'éloquence traditionnelle. De ces deux modèles, l'un propose un renouvellement de la prédication pathétique, enthousiaste et improvisée, adaptée aux esprits du $\mathrm{XIX}^{\mathrm{e}}$ siècle par la forme des raisonnements et des preuves, mais assez traditionnelle quant à la structure du discours; l'autre se dirige vers la pratique de réécriture poétique et politique du message de la Bible tout en rejetant le spectacle de la parole publique - le spectacle de l'éloquence pour inviter à une lecture privée, silencieuse mais non moins subversive des textes sacrés.

\section{RHETORIQUE ET RELIGION}

Dans ses Épîtres, Saint Paul de Tarse soulève le problème de la manière de prêcher au peuple de Dieu le message divin, en insistant sur la «folie» de la prédication et sur la nécessité de changer de langage et de logique pour suivre l'enseignement du Christ. Cette veine paulinienne, contraire au rationalisme ou même logocentrisme gréco-romains qui fondaient les principes de l'éloquence, a trouvé des continuateurs dans la tradition chrétienne (on peut penser à Tertullien et Origène), mais il semble que la doctrine officielle de l'Église catholique se fonde d'avantage sur les acquis de la pensée de saint Augustin, au moins dans le domaine de l'éloquence. Rappelons-le, saint Augustin dans son traité De doctrina christiana a travaillé à une sorte de fusion entre le cicéronianisme romain et la prédication chrétienne.

Orateur lui-même, professeur de rhétorique avant de se convertir au christianisme, saint Augustin ne rejette pas comme incompatibles avec la prédication chrétienne le savoir et la pratique rhétoriques anciens. Au contraire, il adapte les fonctions de l'éloquence païenne à des buts chrétiens; il s'agit toujours d'enseigner le message du Christ (docere), d'émouvoir afin de fléchir les cœurs (flectere) et de plaire aux auditeurs de la parole sacrée (delectare). La mission du prédicateur est de transmettre la parole divine de façon claire et compréhensible, en traduisant des passages obscurs et mystiques de la Bible par des images qui parlent au cœur et à l'imagination, qui peuvent infléchir la volonté des ouailles. Manuel Maria Carrilho a décrit ainsi le projet augustinien d'employer la rhétorique à des fins de la religion en occultant l'héritage purement techniciste de la rhétorique grecque :

On y trouve [dans le De doctrina christiana, $M S$ ] une conception neutre du discours dans la mesure où celui-ci peut aussi bien servir la justice que l'injustice - qui met en valeur de façon très explicite le registre de la compréhension intuitive et du sens commun, plaçant au second plan les techniques rhétoriques et leurs fonctions de médiation herméneutique. L'inventio et l'elocutio se subordonnent aux objectifs de l'accès au sens des Écritures (Carrilho, 1999 : 78-79). 
Augustin christianise le savoir rhétorique antique et consacre le lien entre l'éloquence et la prédication. Ce lien continue au XVII ${ }^{\mathrm{e}}$ siècle français où la domination des règles du bien dire dans la parole ecclésiastique est perceptible à la fois dans la pratique des orateurs sacrés tels que Bossuet, Fléchier ou Massillon, et dans les traités de rhétorique qui soumettent l'éloquence de la chaire aux mêmes préceptes que ceux de l'éloquence du barreau ou de la tribune. Souvent, l'éloquence sacrée ne forme qu'un chapitre des traités plus généraux sur tous les genres rhétoriques, et les auteurs ne font que mentionner la spécificité de la tâche de l'orateur sacré (il doit croire ce qu'il dit, il doit être humble). Mais la manière d'annoncer la parole sacrée ne se différencie guère de celle de laquelle on parle au barreau ou à l'académie. Cette unité stylistique et rhétorique s'étend même plus loin, sur tous les genres littéraires, comme en témoigne l'abbé Maury dans son Essai sur l'éloquence de la chaire publiée pour la première fois en $1777^{1}$ :

Quoique cet ouvrage soit spécialement approprié aux candidats de la chaire, les préceptes de l'art d'écrire, les principes de la composition, les règles du bon goût, et presque toute la théorie de l'art oratoire, s'appliquent également à tous les genres de littérature, et peuvent, pour ainsi dire, intéresser autant nos jeunes écrivains que les orateurs eux-mêmes (Maury, 1828 : xij).

Jusqu'à la fin du XVIII ${ }^{\mathrm{e}}$ siècle, il y a donc une certaine unité au sein de la république des lettres, unité assurée par le concept d'éloquence. Cependant, à l'époque romantique, l'idéal de l'art de bien dire à la chaire est récusé entre autres à cause du souvenir révolutionnaire qui a monopolisé l'éloquence à la fin $\mathrm{du} \mathrm{XVIII}^{\mathrm{e}}$ siècle et qui l'a fait basculer du domaine religieux dans le domaine politique $^{2}$. Il faut souligner que la crise de l'Église après la Révolution, l'entrée en siècle de "l'indifférence en matière de religion ${ }^{3}$ ", ainsi que la floraison de différentes sectes à caractère quasi-religieux dans les années 1830 (le saintsimonisme et le fouriérisme) ont fortement marqué la prédication de ces temps-là. Face à la crise de la religion, l'Église catholique se trouve donc dans une position où le besoin de l'apologétique, de la meilleure formation des prêtres, ainsi que de l'enseignement le plus basique du catéchisme, sont très sensibles. De plus, le sacerdoce ecclésiastique au début du $\mathrm{XIX}^{\mathrm{e}}$ siècle se trouve assez fortement contrebalancé par un autre pouvoir à caractère quasi-religieux, à savoir

${ }^{1}$ Comme l'indique Isabelle Martin, l'Essai a connu plusieurs publications : les éditions de 1782, 1785, 1804, 1805 n'apportent pas de grands changements dans le texte de l'Essai. Par contre dans celle de 1810 Maury intercale dix-sept nouveaux chapitres. Nous utilisons l'édition de 1828 qui reproduit celle de 1810 (Martin, 2002 :127).

${ }^{2}$ Pour d'autres raisons de la condamnation de l'éloquence à la charnière des XVIII $^{\mathrm{e}}$ et XIX ${ }^{\mathrm{e}}$ siècles, voir : Guilhaumou, $2002:$ 233-234 et Sukiennicka, $2015: 337-338$.

${ }^{3}$ C'est le titre d'un essai de Félicité de Lamennais, paru en 1823. 
celui du "Poète », doté d'un «sacerdoce » laïque nouveau, analysé par Paul Bénichou et José-Luis Diaz (Bénichou, 2004, Diaz, 2007). Les auteurs des traités de rhétorique sacrée concertent une réponse angoissée à la nouvelle esthétique qui mine les fondements de leur pouvoir spirituel fortement ébranlé par la Révolution. Les auteurs tels que l'abbé Vêtu, l'abbé Guillon ou l'abbé Morel sont très méfiants de la nouvelle esthétique romantique, jugée trop extravagante, excessive, frénétique, impropre à provoquer une véritable piété chrétienne. Ils déconseillent aux jeunes séminaristes la lecture de Hugo ou même de Lamartine, dont la religiosité ne leur semble pas suffisamment orthodoxe. Selon l'abbé Hamon, les romantiques "s'abandonnent aux élans de l'imagination, chargent tout ce qu'ils disent d'ornements, d'images, de grands mots jetés à profusion, de néologismes, de constructions forcées, et d'idées vagues et sans précision » (Bowman, $1980: 64)$.

Cette méfiance va restreindre l'influence du romantisme sur la prédication dans les années 1830 et 1840, et surtout sur son élaboration théorique. Pourtant, la nouvelle esthétique laisse sa trace dans la pratique de l'improvisation à la chaire, dans l'élaboration de genres sacrés nouveaux, dans l'abandon de la pratique des divisions rhétoriques au sein du discours, et enfin, dans le langage employé par les orateurs sacrés. Cette problématique apparait lors de la « bataille romantique de l'éloquence sacrée » provoquée par le cycle de conférences de Lacordaire données à la cathédrale de Notre-Dame dès 1835.

\section{LACORDAIRE}

La carrière oratoire d'Henri Lacordaire commence très tôt : déjà au collège, chaque année il remportait les prix d'éloquence et éblouissait ses camarades et professeurs par son style, sa verve et son action oratoire, comme en témoignait Prosper Lorain, un ami de collège de Lacordaire :

Sa langue était merveilleuse de coloris et d'éclat, ses phrases pleines d'éclairs, remplies d'agilité, de ressources inattendues, de souplesse et de saillies. La voix était nette, vibrante, frémissante, haletante, s'enivrant d'elle-même, n'écoutant qu'elle seule et s'abandonnant sans réserve et sans contrainte à la verve intarissable de la plus riche nature (Favre, $1906: 27$ ).

À cette époque-là, Lacordaire, n'ayant pas encore cette foi qui le poussera plus tard à devenir prêtre, ne jurait que par Voltaire et par Rousseau. Il a décidé de choisir la carrière d'avocat où son talent d'orateur pouvait prendre son plein essor. Effectivement, quelques années plus tard, ayant été admis au barreau de Paris, Lacordaire a été salué pour son talent par le plus fameux avocat libéral de l'époque, Pierre-Antoine Berryer. Âme tourmentée, vite déçue par la sécheresse 
des questions légales et nourrie par la lecture de Chateaubriand et de Lamennais, Lacordaire découvre en lui une vocation religieuse et décide d'entrer au séminaire à Issy, où de nouveau il surprend ses professeurs par son aptitude à parler et à raisonner sur les plus fines questions théologiques. Pourtant, les biographes de Lacordaire sont d'accord pour admettre que malgré sa conversion d'avocat en prêtre, il ne change pas de manière de parler que l'on peut qualifier de narcissique ( «la voix [...] s'enivrait d'elle même »), mondaine et surtout profane :

Content de sa première méthode, il y restera fidèle dans l'exercice de son ministère religieux et 1 'avocat parlera encore sous la robe du prêtre et du dominicain. Défenseur ardent de la cause de l'Église, il prononcera des discours qu'on pourrait appeler des plaidoyers en faveur des droits de Dieu et des âmes : et il les donnera à des auditeurs qu'il regardera moins comme des fidèles que comme les membres d'un vaste jury, remplissant la nef des cathédrales et invité à émettre une sentence et un jugement (Favre, $1906: 35)$.

Favre souligne la présence du modèle judiciaire dans l'éloquence de Lacordaire: il défend la cause de Dieu comme un avocat défendrait son client. L'orateur exagère les traits de la prédication traditionnelle (cf. Maury) : il rend encore plus profane le ministère du prédicateur. D'ailleurs, il sera souvent jugé dans la presse non pas sous l'angle de sa fidélité à la doctrine, mais sous l'angle de son habileté oratoire ${ }^{4}$. Dans ses discours, il raisonne moins sur le dogme et la métaphysique en donnant avantage à des exemples et preuves historiques, psychologiques et philosophiques. Ainsi par exemple, la treizième conférence s'ouvre par un raisonnement analogique qui surprend par sa froideur : "Toute science s'apprend par l'étude des phénomènes qui ressortent de son objet. Par conséquent la science religieuse s'apprend par l'étude des phénomènes religieux » (Lacordaire, 1853 : 255). En rompant avec le vieux moule ecclésiastique, Lacordaire commence ses discours non pas par un fragment du texte saint à commenter, mais par une formule philosophique ou sapientielle qui pourrait frapper ses auditeurs incrédules du XIX ${ }^{\mathrm{e}}$ siècle (interpellés, ce qui est significatif, par une simple formule laïque de « Messieurs »). Pour plaire davantage à son public, Lacordaire évite les divisions et subdivisions rhétoriques artificielles qui refroidissent le discours. Il n'y a donc plus nécessairement d'exorde méthodique où l'orateur annonce son sujet; pareillement, la péroraison ne résume plus les points essentiels du développement. Le moule rhétorique est sensiblement relâché. Sa parole coule librement et ne craint pas la digression. Sainte-Beuve soulignait déjà la nouveauté du style de Lacordaire : selon lui, il est " plus de ceux qui relèvent et rehaussent la tradition que de ceux qui la soutiennent»

\footnotetext{
${ }^{4}$ Sainte-Beuve écrivait : «Il est assurément le prédicateur de nos jours qui, aux yeux de ceux qui observent et admirent plus encore qu'ils ne croient, se montre à la plus grande hauteur de talent » (Sainte-Beuve, $1849: 221-222$ ).
} 
(Sainte-Beuve, 1849 : 221). En effet, Sainte-Beuve considérait Lacordaire comme le représentant du romantisme en prédication :

Les Conférences de l'abbé Lacordaire ont un caractère qui ne les rattache à rien de ce qui est réputé classique en ce genre, mais qui est singulièrement approprié à l'auditoire de ce temps-ci. [...] Il faut donc reconnaître que la forme de l'abbé Lacordaire est neuve, et même romantique si l'on veut [...]. Cette école hardie et brillante n'avait point suscité jusque-là son prédicateur, et c'est en l'abbé Lacordaire qu'il s'est rencontré (SainteBeuve, $1849: 227-228)^{5}$.

Comme les romantiques - au moins ceux avec lesquels Sainte-Beuve, auteur du célèbre poème «Les Rayons jaunes », s'identifie, c'est-à-dire les partisans de l'école du désenchantement - Lacordaire comprend la crise moderne de la foi. Comme les romantiques, il refuse de recourir à l'ancienne rhétorique. Lacordaire le dit lui-même en ouverture du tome de ses conférences à travers une figure qui conjugue la captatio benevolentiae et l'humilité : «Il ne s'agit pas de suivre les règles de la rhétorique, mais de faire connaître et aimer Dieu, ayons la foi de saint Paul, et parlons le grec aussi mal que lui » (Lacordaire, 1853 : 10-11). Autre trait éminemment romantique : à la chaire, Lacordaire ne lit pas, il improvise, ce qui va contribuer à cette allure de passion et de vie qui a été tellement appréciée par le public des conférences. L'orateur suivait en cela les intuitions de l'abbé Maury qui critiquait les divisions rhétoriques en insistant sur l'importance de l'improvisation à la chaire. La langue des conférences se distingue par la simplicité et le naturel ; plus de style exclusivement noble et soutenu, les expressions familières et triviales entrent souvent dans les discours de Lacordaire.

En outre, comme le remarquait Sainte-Beuve, le prêtre avait une rare faculté de parler à la jeunesse du $\mathrm{XIX}^{\mathrm{e}}$ siècle; sa sensibilité pouvait se traduire par des images poétiques inspirées par la lecture de Chateaubriand ou de Lamartine. Lacordaire a recours à des expressions propres à la nouvelle sensibilité romantique, quelquefois un peu larmoyante :

Désormais l'Église pouvait aller avec confiance conquérir l'univers, car il y a des larmes dans tout l'univers, et elles nous sont si naturelles, qu'encore qu'elles n'eussent pas de cause, elles couleraient sans cause, par le seul charme de cette indéfinissable tristesse dont notre âme est le puits profond et mystérieux (Lacordaire, $1853: 47$ ).

Sainte-Beuve soulignait aussi la chaleur et l'onction de la parole de Lacordaire, la «nouveauté et souvent le bonheur de l'expression, la vivacité et l'imprévu des mouvements, l'éclat et l'ardeur de la parole par l'imagination et

\footnotetext{
${ }^{5}$ Un autre passage : « Il a du clairon dans la voix, et l'éclair du glaive brille dans sa parole. Il possède l'éloquence militaire appropriée à des générations qui ont eu Chateaubriand pour catéchiste et qu'a évangélisées Jocelyn après René » (Sainte-Beuve, $1849: 232$ ).
} 
même la poésie qui s'y mêlent » (Sainte-Beuve, 1849 : 221) qui faisaient de lui un orateur proprement romantique. Même si son argumentation paraît parfois lacunaire, au dire de l'auteur des Causeries, il enflammait l'auditoire de ses conférences par sa chaleur de son style pittoresque et son imagination poétique (Sainte-Beuve, 1849 : 229).

Il n'y a pas que le style de ses discours, c'est aussi son action oratoire qui attirait l'attention de tous les auditeurs de Lacordaire. Évoquons seulement quelques descriptions de ce qui s'est malheureusement perdu pour toujours et qui n'est reconstituable dans l'imagination que grâce à des témoignages d'époque. Voici un extrait de la revue La Dominicale, qui décrit en 1835 les effets des premières conférences à Notre Dame :

Ces conférences eurent un plein succès. [...] II y avait tant de douceur, de pureté, de tristesse et de mélancolie sur sa physionomie, que les auditeurs y mirent de 1 'intérêt dès le début. Sa parole est douce, ses accents persuasifs [...]. Rien d'apprêté, rien de solennel. Cependant, peu a peu, la timidité s'évanouit, les forces se raniment, l'accent devient plus ferme; au bout de quelques minutes, chacun sent que cette nature frêle a une diction nette, précise, une manière d'exposer élégante, qui annonce l'orateur (Favre, 1906 : 143).

Lacordaire a mis à profit son physique avantageux pour un orateur de la chaire : sa physionomie était pure, angélique mais aussi passionnée ; sa voix était frêle, mais elle se haussait jusqu'au registre énergétique de la persuasion. Et voici encore un autre témoignage sur les effets de son action oratoire, cette fois-ci tiré de La vie de Lacordaire par Joseph Théophile Foisset où l'auteur commente l'effet des conférences au collège Stanislas :

On courait, on se pressait dans la petite chapelle du collège Stanislas pour entendre cette parole vivante, soudaine, palpitante, jaillissant de l'âme et allant à 1'âme ; parole pleine d'imprévu, de saillies, d'élan, toute de flamme, ardente, impétueuse, étincelante, émouvante surtout au-delà de toute idée : une voix parfois déchirée et déchirante, qui faisait vibrer à un point qu'on ne saurait dire toutes les fibres de la nature humaine (Foisset, $1870: 293)$.

Pour résumer, Lacordaire a fait revivre la légende de la langue naturelle, pleine de simplicité et d'émotion; il se servait non pas d'une éloquence sclérosée et scolaire, mais au contraire appropriée, selon les témoignages d'époque, à l'esprit du XIX ${ }^{\mathrm{e}}$ siècle, "jeune, svelte, hardie » (Sainte-Beuve, 1849 : 227), ne craignant pas de « saillir la robe blanche du prêtre » par un mot impropre ou une tournure familière. Dans son article, Sainte-Beuve a placé Lacordaire dans la suite des grands orateurs du XVII ${ }^{\mathrm{e}}$ siècle: de Bossuet, Bourdaloue et de Massillon. Mais Lacordaire a modifié cette tradition par une touche romantique, par l'improvisation, par le fréquent appel aux passions, par des images poétiques incrustées dans cette langue pourtant simple et naturelle. La 
conjonction d'un langage personnel, d'un certain philosophisme et de la mélancolie devait plaire aux romantiques désenchantés. En effet, Lacordaire utilise un modèle de persuasion qui parfois traduit nettement sa provenance profane et son voisinage avec l'éloquence du barreau, ce qui est visible surtout dans la forme de l'argumentation matérialiste (à la manière de Condillac ${ }^{6}$ ) de son projet apologétique et ce qui, par ailleurs, lui a été reproché par l'abbé Guillon et quelques autres auteurs qui combattaient toute résurgence de l'esprit du XVIII ${ }^{\mathrm{e}}$ siècle.

\section{LAMENNAIS}

Une autre critique de ce modèle profane de la prédication viendra du côté de l'ancien ami de Lacordaire, son aîné d'une vingtaine d'années, fondateur du journal catholique et libéral Avenir, Félicité de Lamennais. Le parcours idéologique de Lamennais depuis le catholicisme contre-révolutionnaire jusqu'au libéralisme catholique en rupture totale avec le sacerdoce romain mériterait certainement une exposition plus longue ${ }^{7}$. Ici, nous allons nous concentrer seulement sur la critique mennaisienne de l'éloquence et sur sa recherche d'un autre discours religieux approprié au XIX $\mathrm{X}^{\mathrm{e}}$ siècle.

Selon Lamennais, l'éloquence de la chaire, telle que l'ont pratiquée Lacordaire ou même Bossuet et Fénelon, est trop profondément ancrée dans le spectacle de la parole, dans les vains effets du geste et de la voix. Selon lui, l'éloquence se réduit à quelques effets de manche qui ne sont pas aptes à convertir qui que ce soit. Déjà en 1808 il écrivait :

Au lieu [des] discours nourris de la substance du dogme, dont les orateurs du siècle précédent ont laissé de si magnifiques modèles, l'on n'entendait presque plus dans la chaire chrétienne que de vagues et froides amplifications de morale, où à peine daignait-on, de loin en loin, citer l'Écriture. On eût dit que les ministres de Jésus-Christ rougissaient de son Évangile, et que la simplicité de ce livre divin eût déparé l'élégance, et, pour ainsi dire, humilié l'orgueil de leurs phrases académiques (Lamennais, 1837 : 51).

Lamennais met en doute non seulement l'efficacité de cette éloquence d'apparat, pompeuse et théâtralisée, contraire à l'esprit de la simplicité évangélique, mais aussi le principe de la relation entre l'orateur et l'auditeur de la chaire (Labouret, 2009: 44). La philosophie mennaisienne de la parole divine transmise et enseignée aux hommes exclut en quelque sorte la communication

\footnotetext{
${ }^{6}$ Parmi d'innombrables exemples, citons celui-ci : «Messieurs, la foi est d'abord un acte d'intelligence. L'intelligence est la faculté de recevoir et de combiner les idées ; les idées sont les lois ou les rapports éternels des choses, en tant qu'ils sont aperçus de l'esprit » (Lacordaire, $1853: 257)$.

${ }^{7}$ Pour plus de précisions, voir Bénichou, 2004 : 557-600.
} 
directe ; elle doit passer par cette absence ou cet éloignement qui fondent l'expérience d'écriture et de lecture. Le cliquetis de la chaire qui agit momentanément sur les âmes des fidèles, émeut et plaît, certes, mais il ne fait pas accéder au mystère de la foi et ne donne pas la vraie consolation chrétienne. Par là, curieusement, Lamennais rejoint un topos de la critique romantique de l'éloquence classique : elle est futile et périssable ${ }^{8}$. Le discours religieux, tel qu'il le conçoit Lamennais, se construit dans un langage opposé à la clarté oratoire et au style élégant de la déclamation. Il faut trouver un style plus dense, plus transcendant et qui permettrait au lecteur d'effectuer la recherche plus approfondie du sens spirituel de la parole écrite: "Tout l'art d'écrire consiste à montrer sous les mots ce qui n'est point en eux, à faire entendre ce qu'on n'a ni réellement dit ni pu dire, à forcer le lecteur de produire en soi l'idée et le sentiment que l'on veut lui communiquer : et l'on voit que ce problème est au fond celui de tous les arts » (Lamennais, $1846: 451$ ). L'art d'écrire nécessite donc toute une rhétorique du non-dit qui soit aussi celle de l'exhortation.

Nous avons un exemple de cette littérature nouvelle dans les Paroles d'un croyant (1834), où le prophétisme, le symbolisme dense et exigeant, côtoient toutefois une rhétorique féroce et combative, très éloignée de celle qu'on aurait pu admettre dans la chaire. Enracinées dans l'esthétique du fragment, les images et les citations de la Bible s'entrecroisent avec les incitations directes à la révolte politique contre les nouveaux tyrans qui rendent impraticable le message christique de l'égalité et de fraternité des chrétiens. Prenant un accent rousseauiste, Lamennais tisse sur le patron biblique des généalogies du peuple au $\mathrm{XIX}^{\mathrm{e}}$ siècle, il raconte l'origine des maux sociaux : la source de la nécessité du travail, de l'exploitation, de l'oppression politique et de la pauvreté. La préface du livre est constituée d'une adresse directe au peuple (on est loin de « Messieurs » de Lacordaire), ce nouveau sujet de la politique du XIX ${ }^{\mathrm{e}}$ siècle :

\section{AU PEUPLE}

Ce livre a été fait principalement pour vous ; c'est à vous que je l'offre. Puisse-t-il, au milieu de tant de maux qui sont votre partage, de tant de douleurs qui vous affaissent sans presque aucun repos, vous ranimer et vous consoler un peu (Lamennais, $1897: 3$ ).

Au nom de l'Évangile, Lamennais remet en cause plusieurs fondements de la société de la première moitié du $\mathrm{XIX}^{\mathrm{e}}$ siècle, à savoir le régime monarchique, la propriété privée et l'hérédité. Lamennais dénonce aussi la soumission du peuple aux tenants du capital exploitant les forces vives de la société ${ }^{9}$. Aux

\footnotetext{
${ }^{8}$ Cf. par exemple Victor Hugo dans le Dernier Jour d'un Condamné (Hugo, 2000 : 147-149).

${ }^{9}$ Le chapitre VIII propose une genèse de l'appauvrissement du peuple exploité par son oppresseur qui demande toujours plus de travail pour une rémunération qui diminue au rythme du
} 
alentours de 1830, l'air n'est plus à la contre-révolution et aux tentatives de restauration du vieux régime politique. Lamennais prend un accent socialiste et il transforme son éloquence religieuse en une parole politique.

Dans un langage à la fois pittoresque et onirique - les motifs du sommeil et de la vision prophétiques reviennent plusieurs fois au cours de l'ouvrage Lamennais prédit une nouvelle apocalypse qui va refonder l'ordre politique :

Fils de l'homme, monte sur les hauteurs, et annonce ce que tu vois. Je vois à l'horizon un nuage livide, et autour une lueur rouge comme le reflet d'un incendie. Fils de l'homme, que vois-tu encore ? Je vois la mer soulever ses flots, et les montagnes agiter leurs cimes. Je vois les fleuves changer leurs cours, les collines chanceler, et en tombant combler les vallées. Tout s'ébranle, tout se meut, tout prend un nouvel aspect [...] Je vois les peuples se lever en tumulte et les rois pâlir sous leur diadème. La guerre est entre eux, une guerre à mort (Lamennais, $1879: 9$ ).

De diverses formes littéraires composent le recueil des Paroles : depuis cette forme dialoguée citée au-dessus, par les formes purement argumentatives, en passant par la parabole et l'allégorie - comme dans le récit sur les origines de l'inégalité dans la société, raconté à aide de l'histoire du Serpent et des rois ${ }^{10}$ jusqu'à la prière et au prône qui incite directement le peuple à la révolte politique. Parfois, Lamennais prend un ton plus pamphlétaire en désignant clairement l'ennemi du peuple qu'il faut combattre :

Après cela, ils déchaîneront contre vous leurs satellites ; ils feront bâtir des prisons sans nombre pour vous y enfermer ; ils vous poursuivront avec le fer et le feu ; ils vous tourmenteront et répandront votre sang comme l'eau des fontaines. Si vous n'êtes pas résolus à combattre, sans relâche, à tout supporter sans fléchir, à ne jamais vous lasser, à ne céder jamais, gardez vos fers et renoncez à une liberté dont vous n'êtes pas dignes (Lamennais, 1879 : 66-67).

caprice du capitaliste. Lamennais met aussi en doute l'adage qui veut que la pauvreté du peuple vienne de la volonté de Dieu. Selon l'auteur, elle est le résultat de la convoitise des méchants (Lamennais, 1879: 29). Ainsi Lamennais révise le dogme d'un certain néocatholicisme qui affirmait « la racine surnaturelle et le caractère nécessairement absolu du pouvoir politique, la prééminence de la religion sur la loi qui en dérive, du dogme révélé sur la raison individuelle » (Bénichou, 2004 : 558).

${ }^{10}$ Les hommes à l'origine vivaient en frères et ils étaient heureux. Un jour, le Serpent a parlé à l'oreille des hommes et ils se sont proclamés rois (Lamennais, 1879 : 12). Désormais, c'est fut la Peur et l'oppression qui régnaient sur terre : «Et ceux qui avaient dit : 'Nous sommes rois' prirent un glaive, et suivirent la Peur de cabane en cabane. Et il se passa là des mystères étranges ; et il y eut des chaînes, des pleurs et du sang » (Lamennais, 1879 : 13). Selon Lamennais, la monarchie provient de Satan et n'est qu'une oppression du peuple. Mais les rôles seront inversés : les rois seront damnés lors du Jugement Dernier pour ne pas avoir aimé leurs frères, c'est-à-dire toute l'humanité, les diverses nationalités confondues. 
Une autre allégorie invite directement les lecteurs à penser la révolution socialiste. Lamennais compare l'organisation sociale des hommes à celle des animaux et juge celle-là nettement meilleure :

Pourquoi les animaux trouvent-ils leur nourriture, chacun suivant son espèce ? C'est que nul parmi eux ne dérobe celle d'autrui, et que chacun se contente de ce qui suffit à ses besoins. Si, dans une ruche, une abeille disait : 'Tout le miel qui est ici est à moi', [...] que deviendraient les autres abeilles ? La terre est comme une grande ruche, et les hommes sont comme des abeilles. Chaque abeille a droit à la portion de miel nécessaire à sa substance, et si, parmi les hommes, il en est qui manquent de ce nécessaire, c'est que la justice et la charité ont disparu au milieu d'eux (Lamennais, 1879 : 17).

Dans un autre passage, Lamennais tente d'assurer le peuple au sujet de la Providence divine en contant une histoire des oisillons délaissés par leur mère mais dont s'occupe un oiseau du nid voisin. La simplicité et la candeur de ces paraboles contraste avec les fragments plus pessimistes et catastrophiques cités ci-dessus. En effet, hormis des incitations à la révolte politique, Lamennais transmet aussi un message de l'amour fraternel, de la nécessité de l'union de toutes les nations qui fonderaient une nouvelle humanité après la fin du règne de Satan (Lamennais, 1879: 32-36). Lamennais a souvent recours à de courtes narrations, dont le sens est parfois donné explicitement de façon très succincte ( «L voyageur c'est l'homme, le voyage c'est la vie, le rocher, ce sont les misères qu'il rencontre à chaque pas sur sa route »; Lamennais, 1879 : 23), ou dont le sens est caché aux lecteurs qui doivent eux-mêmes déchiffrer les symboles du récit (par exemple dans le chapitre XIII). Cette multiplicité des formes littéraires, animées par un esprit de révolte contre l'ordre politique établi, nourries par de nombreuses citations de la Bible sorties de leur contexte historique et placées dans celui du $\mathrm{XIX}^{\mathrm{e}}$ siècle, bouleversent profondément la rhétorique religieuse pratiquée à l'époque romantique. La nouveauté la plus frappante, c'est le mélange de l'éloquence sacrée et de l'éloquence de la tribune, procédé qui va être largement utilisé dans de différents écrits utopistes tout au long XIX ${ }^{\mathrm{e}}$ siècle. Si la jonction du religieux et du politique n'apparaît pas, théoriquement parlant, inconcevable dans les traités rhétoriques néoclassiques (cf. Maury), la pratique du discours socialiste bouleverse à tel point la tradition rhétorique par sa force, sa rudesse et son style prophétique féroce et sanglant que l'on peut parler d'une véritable révolution dans la pratique de la parole sacrée.

Pour conclure, les différences dans l'appréciation du discours religieux chez Lacordaire et Lamennais peuvent s'expliquer dans une certaine mesure par leur rapport à l'Église catholique romaine. Lacordaire, après les encycliques Mirari vos de 1832 et Singulari Vos de 1834 condamnant les idées politiques de Lamennais, a juré une entière obéissance au Saint Siège. Par la suite, dans ses conférences, il souligne l'importance de cette institution et de sa mission civili- 
satrice et politique au $\mathrm{XIX}^{\mathrm{e}}$ siècle. Lacordaire parle du centre de l'Église et au nom de son autorité ; il assimile le rôle de l'Église à la persuasion pacifique et l'oppose à tout combat physique, violent, contre les gens et les régimes. Lamennais au contraire s'éloigne de ce centre, il devient réfractaire et solitaire dans sa quête religieuse et politique. Il choisit une parole prophétique qui se situe entre les deux extrêmes: entre le cri de la révolte et le silence de l'expérience intérieure du divin. De l'autre côté, le parcours et le succès de Lacordaire à l'époque des conférences de Notre-Dame résument en quelque sorte le statut de la rhétorique dans le romantisme : en effet, ils ne sont aucunement incompatibles. Le mouvement littéraire des années 1830 constitue un des derniers moments en France ${ }^{11}$ de la gloire oratoire encore assez traditionnelle, qui s'accomplit dans une communication entre l'orateur et le public et qui est toujours fondée sur les mêmes références et les même attentes génériques et littéraires. Si Lacordaire s'attache à remplir dans ses discours surtout la vieille fonction de delectare, Lamennais investit plutôt une tâche plus rude de flectere et de docere tout en inaugurant la recherche d'une autre écriture prophétique, biblique et visionnaire, qui ne craint pas de sombrer dans le militantisme politique, mais qui essaie aussi de décrire dans une poétique renouvelée quelques expériences de « voyance » toute moderne. De plus, sur l'exemple de la pratique de l'éloquence religieuse de Lacordaire et de Lamennais, on observe le processus qu'Alain Vaillant a décrit comme le passage de la littérature-discours à la littérature-texte qui s'accompli au tournant des années 1830 :

Sous les formes les plus variées [...], la littérature a ainsi permis pendant des siècles la sublimation scripturale de la parole : nous appellerons désormais littérature-discours ce premier avatar du système textuel. Mais il est vrai que, à partir du XIX ${ }^{\mathrm{e}}$ siècle $[\ldots]$ cette rhétoricité littéraire a fait l'objet d'une profonde remise en cause. La littérature n'est plus apparue comme un discours donné à entendre (même par le truchement de l'écrit), mais comme un texte à lire, un réseau de signes soumis à l'herméneutique silencieuse du lecteur. Cette nouvelle littérature, que nous nommerons littérature-texte, se marque par l'effacement du modèle discursif et par l'impersonnification de l'écriture (Vaillant, $2010: 255)$.

Avec Lamennais, le modèle de l'éloquence orale est évacué de la production des textes religieux au profit d'une expérience solitaire et taciturne de la lecture. Ce phénomène ne fera que croître en ampleur tout au long du $\mathrm{XIX}^{\mathrm{e}}$ siècle puisque le discours religieux se déplaçait peu à peu de la chaire au livre ${ }^{12}$.

${ }^{11}$ Guilhem Labouret affirme que «le $\mathrm{XX}^{\mathrm{e}}$ siècle n'a pas connu de prédicateurs de renom, tels que le $\mathrm{XIX}^{\mathrm{e}}$ avait pu en connaître. C'est dans la poésie, le théâtre ou l'essai que le discours religieux domine, et non plus dans le sermon » (Labouret, $2009: 39$ ).

${ }^{12}$ L'article s'inscrit dans le cadre du projet financé par le Centre National de la Recherche Scientifique (Narodowe Centrum Nauki) ; le numéro de référence : UMO-2012/05/N/HS2/02744. 


\section{BIBLIOGRAPHIE}

Benichou, PAUl (2004). Les romantismes français. T. 1. Paris : Quarto.

Bowman, Frank PAUl (1980). Le discours de la chaire à l'époque romantique. Genève : Droz.

CARrilho, Manuel Maria (1999). Au-delà du langage. La seconde sophistique et la rhétorique chrétienne. In M. Meyer (eds.), Histoire de la rhétorique des Grecs à nos jours (pp. 73-82), Paris : Le livre de poche.

DiAz, JosE-LuIS (2007). L'écrivain imaginaire. Scénographies auctoriales à l'époque romantique. Paris : Honoré Champion.

FAVRE, JULIEN (1906). Lacordaire orateur. Paris : Poussielgue Librairie.

FoISSET, JosePH THEOPHILE (1870). La vie du révérend père Lacordaire. T. 1. Paris : Librairie Jacques Lecoffre.

GuilHAUMOU, JACQUES (2002). La rhétorique des porte-paroles (1789-1792). In E. Négrel, J. P. Sermain (eds.), Une expérience rhétorique, l'éloquence de la révolution (pp. 221-239). Oxford : Voltaire University Foundation.

Hugo, Victor (2000). Le Dernier Jour d'un Condamné. Paris : Folio.

LABOURET, GUILHEM (2009). Les mutations du discours religieux au XIX ${ }^{\mathrm{e}}$ siècle, Romantisme, $144,39-53$.

Lacordaire, Henri-Dominique (1853). Conférences de Notre Dame. T. 1. Paris: Sagnier et Bray.

Lamennais, Felicite de (1837). Cuvres complètes. T. 6. Paris : Daubrée et Cailleux.

- (1846). Esquisse d'une philosophie. T. 3. Paris : Pagnerre Editeur.

- (1879). Paroles d'un croyant. Paris : Librairie de la Bibliothèque Nationale.

MARTIN, IsABELle (2002). «Usage de la dramaturgie racinienne dans l'Essai sur l'Éloquence de la Chaire de l'abbé Maury ». Dix-septième siècle, 214, 127-136.

MAURY, JEAN-SifReIn (1828). Essai sur l'éloquence de la chaire. Paris : Gayet Libraire.

Sainte-Beuve, Charles-Augustin (1849). Causeries du lundi. T. 1. Paris : Garnier Frères.

SuKIENNICKA, MARTA (2015). «Essai sur l'art oratoire» de Joseph Droz: entre le naturalisme antirhétorique et l'éloquence romantique. Orbis linguarum, 41, 335-344.

Vaillant, Alain (2010). L'histoire littéraire. Paris : Armand Colin. 
